\title{
Pulmonary fibrosis combined with lung cancer following lung transplantation: should we do more?
}

\author{
Guilin Peng ${ }^{1}$, Chao Yang ${ }^{1}$, Mengyang Liu ${ }^{1}$, Weixue Cui ${ }^{1}$, Bastian Grande ${ }^{2}$, Christina Kao ${ }^{3}$, \\ Piergiorgio Sollii ${ }^{4}$, George Makdisi ${ }^{5}$, Xin $\mathrm{Xu}^{1}$, Jianxing $\mathrm{He}^{1}$ \\ ${ }^{1}$ Department of Thoracic Surgery and Organ Transplantation, First Affiliated Hospital of Guangzhou Medical University, Guangzhou, China; \\ ${ }^{2}$ Institute of Anesthesiology, University Hospital Zurich, Zurich, Switzerland; ${ }^{3}$ Section of Pulmonary, Critical Care, and Sleep, Department of \\ Medicine, Baylor College of Medicine, Houston, TX, USA; ${ }^{4}$ Department of Cardio-Thoracic Surgery and Hearth \& Lung Transplantation, IRCCS \\ Azienda Ospedaliero-Universitaria di Bologna, Bologna, Italy; ${ }^{5}$ Division of Cardiothoracic Surgery, University of South Florida, Tampa General \\ Hospital, Tampa, FL, USA \\ Correspondence to: Xin Xu; Jianxing He. Department of Thoracic Surgery and Organ Transplantation, First Affiliated Hospital of Guangzhou Medical \\ University, Guangzhou 510120, China. Email: yichunrenjia@126.com; drjianxing.he@gmail.com.
}

\begin{abstract}
Currently, lung transplantation is the standard of care for patients with end-stage lung disease, with interstitial lung disease (ILD) being the most common reason in the recent years In the other hand, in cases where stage II and III lung cancer have been identified following lung transplantation, long-term survival outcomes are poor when compared to lung cancer patients that have not received a lung transplant because the use of immunosuppressant and the problem of rejection and infection and the treatment of recurrence and so on. However, there is no statistical difference observed in stage I (pT1N0M0) patients. In this paper we report about a patient with ILD receiving left lung transplantation in the early time. A lesion of the right lung which was considered the normal ILD tissue and without enough attention. Post-transplant it showed progress and finally the whole right lung (native lung) was occupied by the tumor. Some ground glass changes could also be found in the transplanted lung several months later. A secondary lung transplant was performed for this patient, and there has been no postoperative recurrence thus far. For lung transplant patients with high-risk factors, effective surveillance methods are required for the early detection of lung cancer.
\end{abstract}

Keywords: Pulmonary fibrosis; lung cancer; lung transplantation; surveillance

Submitted Sep 24, 2020. Accepted for publication Mar 05, 2021.

doi: $10.21037 /$ tlcr-21-46

View this article at: http://dx.doi.org/10.21037/tlcr-21-46

\section{Introduction}

Lung transplantation is a poor treatment option for lung cancer patients, with almost all lung cancers reported in the literature occurring post-lung transplant (1). Lung cancer may originate from either the implanted lung or the native lung. The use of immunosuppressants reduces the lung cancer survival rates of patients that receive lung transplants, and there are currently no effective treatments available (2). In this paper, we report a case of pulmonary fibrosis with lung cancer following single lung transplantation. A secondary lung transplant was performed 8 months later.
We present the following article in accordance with the CARE reporting checklist (available at http://dx.doi. org/10.21037/tlcr-21-46).

\section{Case presentation}

All procedures performed in studies involving human participants were in accordance with the ethical standards of the institutional and/or national research committee(s) and with the Helsinki Declaration (as revised in 2013). Written informed consent was obtained from the patient. 


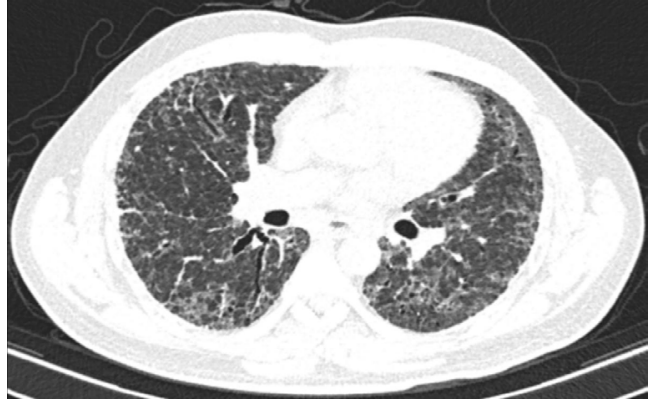

Figure 1 Preoperative chest CT. CT, computed tomography.

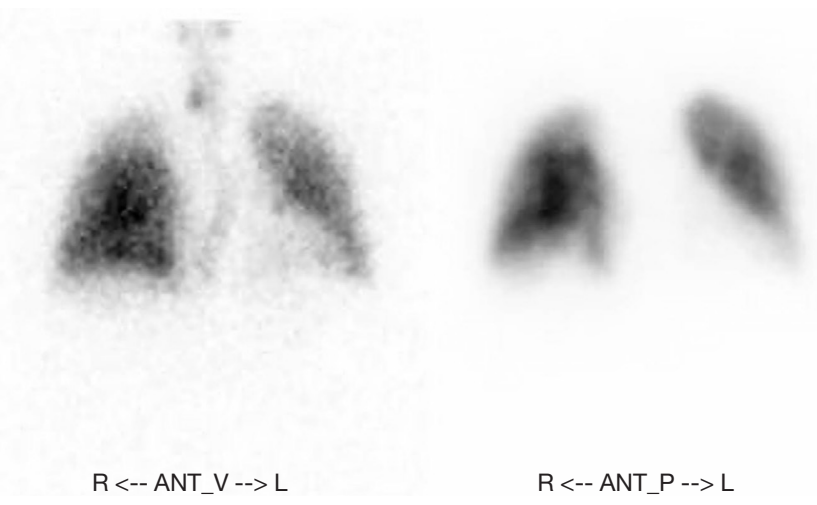

Figure 2 Preoperative pulmonary ventilation-perfusion imaging.

\section{Lung transplant recipient}

The transplant recipient in this study was a 42-year-old man diagnosed with idiopathic pulmonary fibrosis (IPF). He had a history of smoking (approximately 20 cigarettes per day for more than 20 years) and quit smoking shortly before his transplant surgery. The patient had no family history of lung cancer. A computed tomography (CT) scan revealed interstitial pneumonia in both lungs (Figure 1), and small lymph nodes less than $1 \mathrm{~cm}$ in the mediastinum and bilateral hilar. Pulmonary ventilation-perfusion imaging showed $40 \%$ in the left lung and $60 \%$ in the right lung (Figure 2). Before surgery the patient was not suspected of pulmonary hypertension although no right cardiac catheterization has been performed. The carcinoembryonic antigen (CEA) was $18.07 \mathrm{ng} / \mathrm{mL}$ (i.e., $<5 \mathrm{ng} / \mathrm{mL}$ ) prior to surgery. Smoking history and the IPF could both explain slightly raised CEA values.

\section{Lung details: donor 1}

Lung donor 1 was a 45-year-old man who suffered from spontaneous intracerebral hemorrhage. Tracheal intubation has been established for 1 day. He was a non-smoker with no family history of lung cancer. The oxygenation index before procurement was 404 .

\section{Transplant surgery and follow up}

Left lung transplantation was performed in December 2018 without extracorporeal membrane oxygenation (ECMO) support and tracheal extubation in the operating room. Immunosuppressive therapy was initiated with mycophenolate mofetil (CellCept), tacrolimus (Prograf), and methylprednisolone. The patient was transferred to the general ward on Day 10 and discharged on Day 18.

No acute rejection occurred, however pulmonary effusion of the right lung (native lung) increased consistently (Figure 3), and some ground glass changes could be seen in the left lower lobe (of the implanted lung). The size of the lymph nodes remained unchanged. Ultrasound guided bronchoscopic punction showed mucinous adenocarcinoma of the right lung and positron emission tomography CT (PET-CT) scan found 18F-fluoro-2-deoxy-D-glucose (18FDG) uptake of the whole right lung. The maximum standardized uptake value (SUVmax) was 8.5 (Figure 4), and 4.4 in the left lower lobe (Figure 5). We found no uptake in the hilar and mediastinum lymph node, and no distant metastasis. The CEA on the $22^{\text {nd }}$ August 2019 was $30.02 \mathrm{ng} / \mathrm{mL}$.

\section{Lung details: donor 2}

Lung donor 2 was a 34-year-old man who experienced brain trauma. Tracheal intubation was necessary for 6 days. There was no pathogenic bacteria identified in the sputum culture. He was a smoker (approximately 10 cigarettes per day for more than 10 years) but had no family history of lung cancer. The oxygenation index before procurement was $522 \mathrm{mmHg}$.

\section{Transplant surgery}

Right lung transplantation was performed in August 2019 (the left lung was discarded due to severe pulmonary edema). Adenocarcinoma was confirmed following wedge resection. Lobectomy (LLL) and lymph node sampling (Station 11 and 7) were subsequently performed and no lymph node metastasis was confirmed in the frozen section. Immunosuppressive therapy was initiated with 

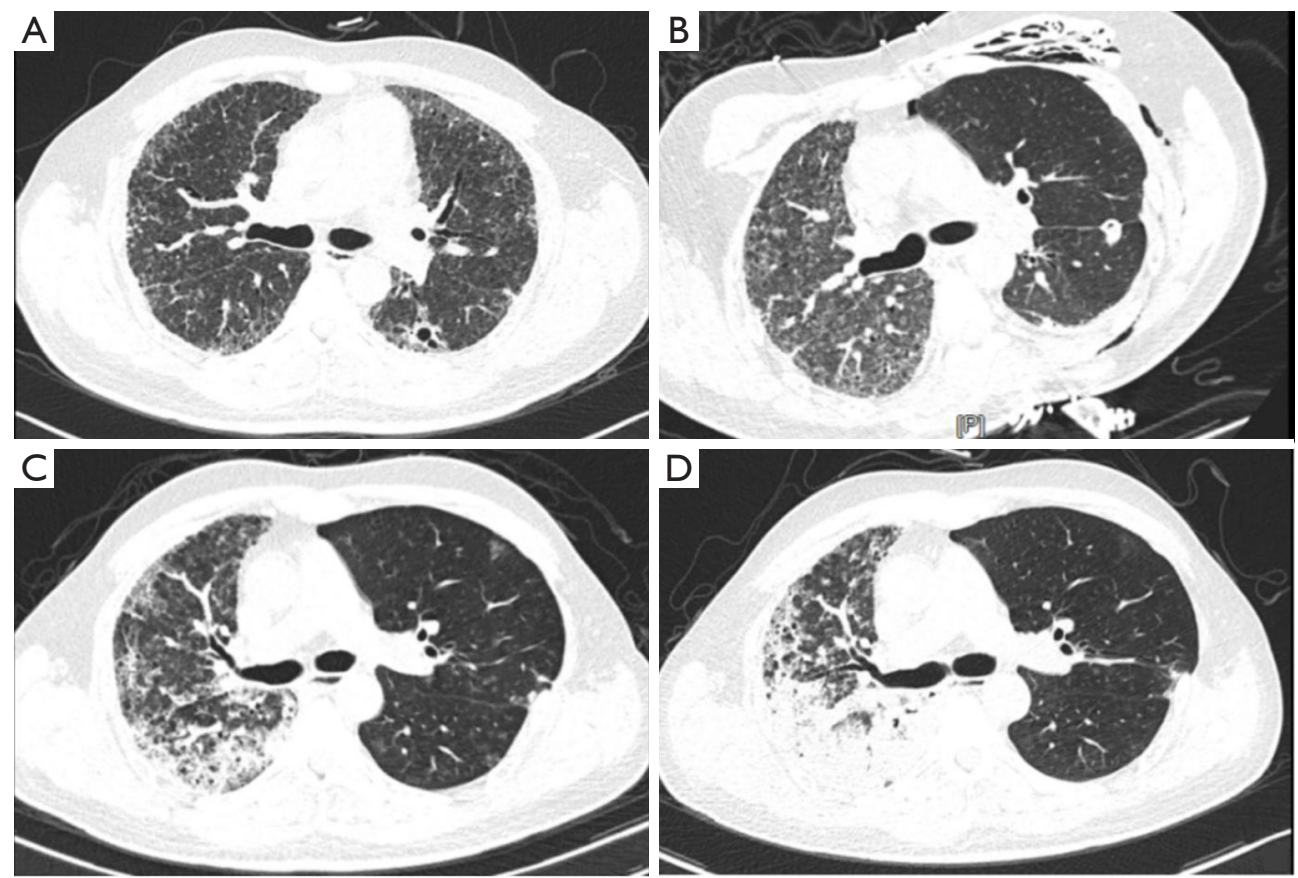

Figure 3 The changes of the native lung (right lung) during the CT follow up. (A) Preoperative chest CT; (B) postoperative chest CT, 4 months; (C) postoperative chest CT, 9 months; (D) postoperative chest CT, 12 months. CT, computed tomography.

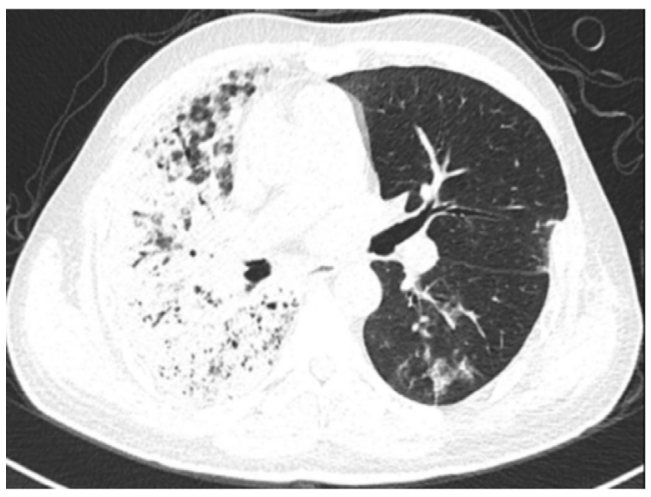

Figure 4 Chest CT prior to the secondary lung transplantation. CT, computed tomography.

mycophenolate mofetil (CellCept), tacrolimus (Prograf)/ cyclosporin A, methylprednisolone. The patient was transferred to the general ward on Day 8 and discharged on Day 34.

\section{Follow-up}

Gene detection was performed for this patient on the $16^{\text {th }}$ September 2019 and Kirsten Rat Sarcoma Viral Oncogene
Homolog (K-RAS) G12A 14.34\% was identified. The CEA on $20^{\text {th }}$ March 2020 was $2.34 \mathrm{ng} / \mathrm{mL}$ (Figure 6). Thus far, no postoperative recurrence has been found for 1 year.

\section{Discussion}

Although the first case of lung transplantation was performed in 1963 in an advanced bronchial carcinoma patient with respiratory failure [i.e., chronic obstructive pulmonary disease (COPD)] (3), lung transplantation is a poor treatment option for lung cancer patients. It was previously employed as a management strategy for bronchioloalveolar carcinoma (BAC), which presented as a localized lesion (4). However, the survival of these patients is poor, owing primarily to the use of immunosuppressants. In recent years, IPF has been the major basis for lung transplantation, with lung cancer occasionally occurring postoperatively. A large data analysis study of postoperative lung cancer from Toronto General Hospital and the United Network for Organ Sharing (UNOS) Registry System found that the postoperative morbidity of lung cancer was $0.5 \%$ and $0.13 \%(5,6)$. Lung cancer may originate in either the implanted lung or the native lung. Risk factors for the development of postoperative lung cancer include: IPF, 


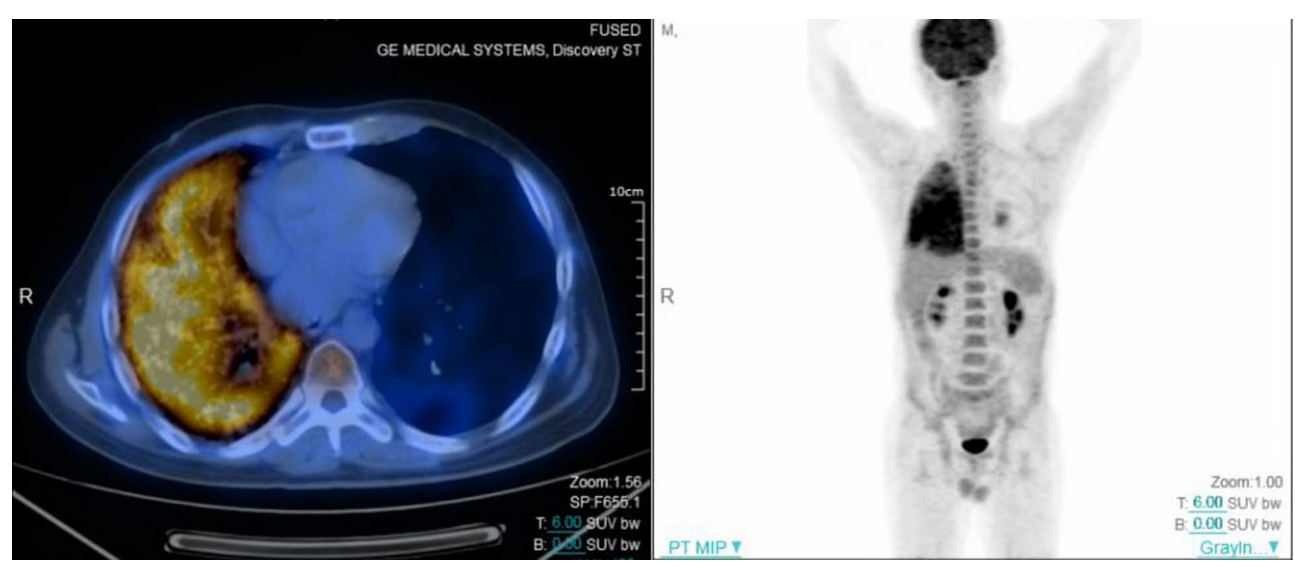

Figure 5 PET-CT prior to the secondary lung transplantation. PET-CT, positron emission tomography computed tomography.

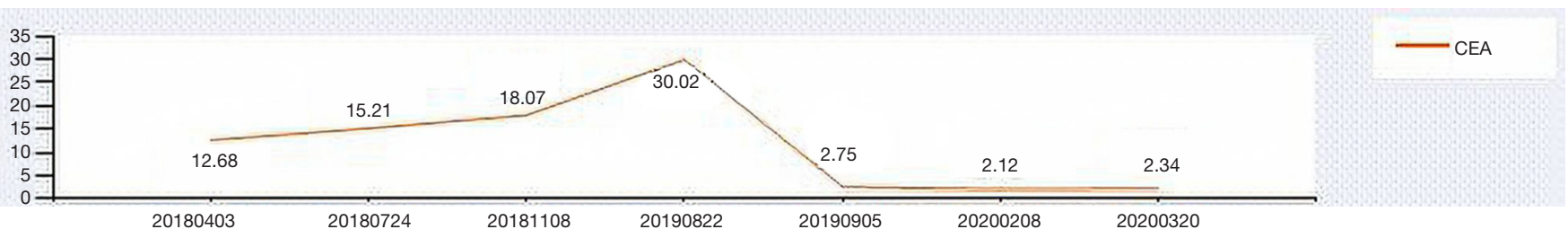

Figure 6 The CEA changing curves. CEA, carcinoembryonic antigen.

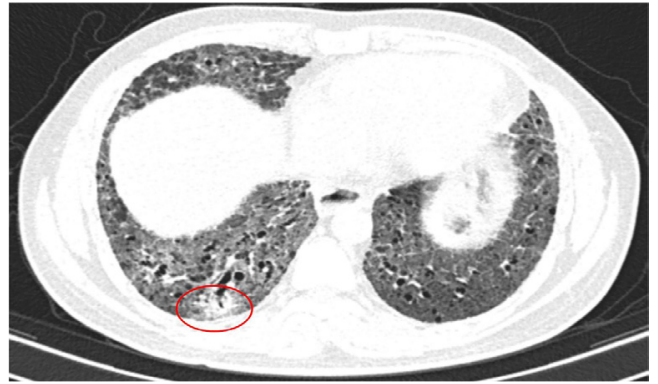

Figure 7 The suspicious lesion of the right lower lobe.

a history of smoking, older age (either the donor or the recipient), male gender, prolonged immunosuppression, and single lung transplantation (7). Of these factors, IPF represents the highest risk for lung cancer, with a 4.96 rate ratio for developing lung cancer in IPF patients compared to the general population (8).

Some lung cancer patients cannot be identified preoperatively due to the following factors: 1 . Loss of donor information: insufficient smoking history, incomplete family lung cancer history, and no CT scan of the donor, which may increase the risk of missed diagnosis of small nodules in the lung; 2. with the smoking and family history of lung cancer of the recipient, lung cancer could be identified after the use of immunosuppressants; 3 . tumors from the bronchial mucosa could not be found previously, and may postoperatively spread to the main bronchus of the implanted lung.

In our centre, CT scans, as well as serological indicators of CEA, SCC, and NSE are routinely tested. Frozen sectioning is recommended for suspicious nodules in the lung during procurement. In this patient, it seems that the lesion of the right lower lobe (Figure 7) was suspicious before the first lung transplantation but without enough attention. In fact, diagnosis is often difficult in IPF patients due to the diffuse lung abnormalities, and small nodules may even be considered as fibrotic lesions (9). These suspicious lesions progressed rapidly due to the use of immunosuppressants (Figure 3). Therefore, surveillance of lung cancer in high-risk patients (both the recipient and the donor) is critically important.

The use of immunosuppressants may lead to earlier tumorigenesis; however, a lower dose of immunosuppressants would increase the risk of rejection and immune surveillance failure (10). If nodules are detected at regular follow-ups and are highly suspected to be lung cancer, active approaches to obtain the pathology 
are recommended. Lobectomy and lymphadenectomy are required for Tumor Node Metastasis (TNM) staging and prognostic prediction if the patient in a good condition. de Perrot et al. (11) reported that the 5 -year survival was better in 22 patients with stage I disease compared to 14 patients with stage II-III ( $51 \%$ versus $14 \%$, respectively).

The question remains: what should be done for patients diagnosed with lung cancer post-lung transplantation? Chemotherapy and radiotherapy are not suitable for these patients, due to their immunosuppressed state (12). Immunotherapy is a highly effective treatment with a good response in advanced stage lung cancer in cases with high programmed cell death 1 (PD-1) or programmed death-ligand 1 (PD-L1) expression. However, the use of immunotherapy still lacks advanced clinical data, particularly large sample analyses. Over the past 20 years, targeted therapy has emerged as an effective measure for the treatment of advanced lung cancer patients with epidermal growth factor receptor (EGFR), anaplastic lymphoma kinase (ALK), or ROS proto-oncogene 1 (ROS-1) mutations. Without the significant immunosuppression compared to chemotherapy and radiotherapy, targeted therapy presents a potential option for these patients, although the clinical data is still lacking.

In this study, due to severe pulmonary edema, the patient underwent right lung transplantation and lobectomy (left lower lobe). Unfortunately, this presented a significant risk compared to double lung transplantation. Postoperative K-RAS mutation was found by gene detection. Since there are no targeted drug therapies available, no specific treatment was provided, and patient only received regular follow-ups.

Enhanced postoperative surveillance may help to discover tumors earlier. PET-CT is a useful examination to confirm the metastasis and uptake of the tumor (13), but not for regular screening. Tumor biomarkers (such as CEA, SCC, and NSE) are also useful for preliminary screening, however given their low sensitivity and specificity, these results may confuse clinicians, especially in IPF patients with lung cancer. Therefore, more sensitive surveillance methods are needed, such as tumor methylation and circulating tumor cell (CTC) analysis (14).

\section{Conclusions}

In general, lung transplantation is a poor treatment option for lung cancer patients, as the use of immunosuppressants results in worse long-term survival outcomes. End-stage lung disease patients need to be aware of the risk for posttransplant tumorigenesis, especially in those with highrisk factors (such as a history of smoking in the donor and/ or recipient, a family history of lung cancer, tumor history within the previous 5 years, and IPF). When lung cancer occurs in these patients, irrespective of whether it occurs in the native lung or the implanted lung, pathology and TNM staging are necessary to determine the survival prognosis. Tumor methylation and CTC analysis may be more sensitive methods for earlier detection of lung cancer.

\section{Acknowledgments}

The authors appreciate the academic support from AME Lung Cancer Collaborative Group.

Funding: Medical Science and Technology Research Fund of Guangdong Province (A2019261).

\section{Footnote}

Reporting Checklist: The authors have completed the CARE reporting checklist. Available at http://dx.doi.org/10.21037/ tlcr-21-46

Conflicts of Interest: All authors have completed the ICMJE uniform disclosure form (available at http://dx.doi. org/10.21037/tlcr-21-46). The authors have no conflicts of interest to declare.

Ethical Statement: The authors are accountable for all aspects of the work in ensuring that questions related to the accuracy or integrity of any part of the work are appropriately investigated and resolved. All procedures performed in studies involving human participants were in accordance with the ethical standards of the institutional and/or national research committee(s) and with the Helsinki Declaration (as revised in 2013). Written informed consent was obtained from the patient.

Open Access Statement: This is an Open Access article distributed in accordance with the Creative Commons Attribution-NonCommercial-NoDerivs 4.0 International License (CC BY-NC-ND 4.0), which permits the noncommercial replication and distribution of the article with the strict proviso that no changes or edits are made and the original work is properly cited (including links to both the formal publication through the relevant DOI and the license). See: https://creativecommons.org/licenses/by-nc-nd/4.0/. 


\section{References}

1. Bellil Y, Edelman MJ. Bronchogenic carcinoma in solid organ transplant recipients. Curr Treat Options Oncol 2006;7:77-81.

2. Machuca TN, Keshavjee S. Transplantation for lung cancer. Curr Opin Organ Transplant 2012;17:479-484.

3. Hardy JD, Webb WR, Dalton MI. Lung homotransplantation in man. JAMA 1963;186:1065-74.

4. Arenberg D, American College of Chest Physicians. Bronchioloalveolar lung cancer: ACCP evidence based clinical practice guidelines (2nd edition). Chest 2007;132:306S-13S.

5. De Perrot M, Chernenko S, Waddell TK, et al. Role of lung transplantation in the treatment of bronchogenic carcinomas for patients with end-stage pulmonary disease. J Clin Oncol 2004;22:4351-6.

6. Ahmad U, Wang Z, Bryant AS, et al. Outcomes for lung transplantation for lung cancer in the United Network for Organ Sharing Registry. Ann Thorac Surg 2012;94:93540; discussion 940-1.

7. Mathew J, Kratzke RA. Lung cancer and lung transplantation: a review. J Thorac Oncol 2009;4:753-60.

8. Le Jeune I, Gribbin J, West J, et al. The incidence of cancer in patients with idiopathic pulmonary fibrosis and

Cite this article as: Peng G, Yang C, Liu M, Cui W, Grande B, Kao C, Solli P, Makdisi G, Xu X, He J. Pulmonary fibrosis combined with lung cancer following lung transplantation: should we do more? Transl Lung Cancer Res 2021;10(3):15881593. doi: $10.21037 /$ tlcr-21-46 sarcoidosis in the UK. Respir Med 2007;101:2534-40.

9. Hendriks LE, Drent M, van Haren EH, et al. Lung cancer in idiopathic pulmonary fibrosis patients diagnosed during or after lung transplantation. Respir Med Case Rep 2012;5:37-9.

10. Dickson RP, Davis RD, Rea JB, Palmer SM. High frequency of bronchogenic carcinoma after single-lung transplantation. J Heart Lung Transplant 2006;25:1297-301.

11. de Perrot M, Chernenko S, Waddell TK, et al. Role of lung transplantation in the treatment of bronchogenic carcinomas for patients with end-stage pulmonary disease. J Clin Oncol 2004;22:4351-6.

12. Ajithkumar TV, Parkinson CA, Butler A, et al. Management of solid tumours in organtransplant recipients. Lancet Oncol 2007;8:921-32.

13. Strollo DC, Dacic S, Ocak I, et al. Malignancies incidentally detected at lung transplantation: radiologic and pathologic features. AJR Am J Roentgenol 2013;201:108-16.

14. Frank Breitenbuecher, Sandra Hoffarth, Karl Worm, et al. Development of a Highly Sensitive and Specific Method for Detection of Circulating Tumor Cells Harboring Somatic Mutations in Non-Small-Cell Lung Cancer Patients. PLoS One 2014;9:e85350. 